\title{
Numerical Solutions of a System of ODEs Based on Lie-Trotter and Strang Operator-splitting Methods
}

\author{
Rowa E.E. Omer ${ }^{1, *}$, Eihab B.M. Bashier ${ }^{1}$, Arbab I. Arbab ${ }^{2}$ \\ ${ }^{1}$ Department of Applied Mathematics, Faculty of Mathematical Sciences, University of Khartoum, Sudan \\ ${ }^{2}$ Department of Physics, Faculty of Science, University of Khartoum, Sudan
}

Copyright (C) 2017 by authors, all rights reserved. Authors agree that this article remains permanently open access under the terms of the Creative Commons Attribution License 4.0 International License

\begin{abstract}
In the present paper, we consider a first-order exponential splitting method (or exponential Lie-Trotter splitting) and second order exponential splitting method (or exponential Strang splitting method ) for the Cauchy problem. Then we compare the errors between Lie-Trotter splitting and Strang splitting by discretizing the space into $N$ sub-intervals, and compute the convergence rate for both Lie-Trotter splitting and Strang splitting methods.
\end{abstract}

Keywords Exponential Strang Splitting Method, LieTrotter Splitting, Cauchy Problem, Matrix Problem, ODE System

\section{Introduction}

Operator splitting methods are widely used for numerical solution of partial differential equations. The idea behind an operator splitting method is to split the differential operator into sub-operator having simpler forms, to simplify the solution of the resulting sub-problems. The solution of the primal problem is then obtained from the numerical solutions of the sub-problems [1]. Operator splitting is a popular technique for solving coupled systems of partial differential equations, since complex equation system maybe split into separate parts that are more simpler to get solution. Several operator splitting techniques are found in the literature $[2,3,4,5,6]$. We concentrate on two exponential splitting methods: Lie-Trotter splitting and strang splitting .

Consider the Cauchy problem :

$$
\begin{gathered}
\frac{\partial U(t)}{\partial t}=A U(t)+B U(t), \\
\text { with } t \in[0, T], U(0)=U_{0},
\end{gathered}
$$

whereby, the initial function $U_{0}$ is given and $A$ and $B$ are supposed to be linear operators in the Banach-space $X$ with $A$ and $B: X \rightarrow X$ In real applications the operators corresponds to physical operators such as convec- tion and diffusion operators. Splitting methods assume that the problem can be split into two or more terms [7]. Let $U(t)=e^{(A+B) t} U_{0}$ be the solution of the differential equation (1) with initial value $U(0)=U_{0}$. The problem with computing the solution $U(t)$ is that the exponential mapping may not be computationally obtained or too difficult to evaluate exactly. Therefore, the flow map EXP is often approximated using some numerical method [15]. Distinguishing the different approaches, methods based on exact flows are famous as exponential splitting methods. The splitting methods for ordinary differential equations are accurate schemes [17], which may be used with non-small step sizes. One approach in this direction is the construction of higher order methods for which numerical map $\Phi_{t}$ of (1) satisfies,

$$
\Phi_{t}=e^{(A+B) t}+O\left(t^{p+1}\right)
$$

with $p$ being as high order as possible. A standard technique for obtaining such methods is to compose $\Phi_{t}$ from more than two exponentials. As such, a typical non-symmetric composition method often used is

$$
\Phi_{t}=e^{a_{m} A t} e^{b_{m} B t} \ldots e^{a_{1} A t} e^{b_{1} B t} e^{a_{0} A t} e^{b_{0} B t}
$$

and various approach have been suggested for determining conditions on the free parameters $\left(a_{0}, a_{1}, \ldots, a_{m}\right)$ and $\left(b_{0}, b_{1}, \ldots, b_{m}\right)$ satisfy

$$
\sum_{i=0}^{m} a_{i}=\sum_{i=0}^{m} b_{i}=1
$$

Any exponential operator splitting method involving several compositions can be cast into the following form,

$$
e^{t(A+B)}=\prod_{i=1}^{m} e^{a_{i} t A} e^{b_{i} t B}+O\left(t^{m+1}\right)
$$

where $A, B$ are noncommutative operators, Here $t$ defines equidistant time step $[14,16,18,19]$, and $\left(a_{0}, a_{1}, \ldots, a_{m}\right)$ and $\left(b_{0}, b_{1}, \ldots, b_{m}\right)$ are real numbers, that sum to unity. We apply Lie-Trotter splitting as first order splitting method, and 
Strang splitting as second order splitting method for Cauchy problem and compare the results between the two methods. We will use Matlab to out carry the numerical simulations. The rest of this paper is organized as follows. In Section II, we talked about Lie-Trotter Splitting. In Section III, we talked about Strang Splitting. Section IV presents The Consistency of the operator splitting method in general. Section $\mathrm{V}$ provides the Stability of exponential splitting method. In VI, the results. and finally in VII our conclusions.

\section{Lie-Trotter Splitting}

The Lie-Trotter splitting method for solving Equation (1) can be cast into the general form described by Equation (4) with

$$
\begin{array}{r}
m=1, \quad a_{1}=1, \quad b_{1}=1, \\
\text { or } \\
a_{1}=0, \quad a_{2}=1, \quad b_{1}=1, \quad b_{2}=0
\end{array}
$$

respectively, that is, the first numerical solution is given by

$$
u_{1}=e^{A t} e^{B t} u_{0} \text { or } u_{1}=e^{B t} e^{A t} u_{0}
$$

In this paper, Lie-Trotter splitting is displayed as a method, which solves two subproblems sequentially on subintervals $\left[t^{n}, t^{n+1}\right]$, where $n=0,1, \ldots, N-1, t^{0}=0$ and $t^{N}=$ $T$. The different subproblems are related through the initial conditions. The Lie-Trotter Splitting's algorithm is:

$$
\begin{gathered}
\frac{\partial u(t)}{\partial t}=A u(t), \text { with } t \in\left[t^{n}, t^{n+1}\right], u\left(t^{n}\right)=u_{s p}^{n} \\
\frac{\partial v(t)}{\partial t}=B v(t), \text { with } t \in\left[t^{n}, t^{n+1}\right], v\left(t^{n}\right)=u\left(t^{n+1}\right)
\end{gathered}
$$

for $n=0,1, \ldots, N-1$ where by $u_{s p}^{n}=U_{0}$ is given from (1). The approximated split solution at the point $t=t^{n+1}$ is defined as $u_{s p}^{n+1}=v\left(t^{n+1}\right)$.

\section{Strang splitting method}

Strang splitting method can be cast into the form (4) with

$$
\begin{gathered}
m=2, \quad a_{1}=a_{2}=\frac{1}{2}, \quad b_{1}=1, \quad b_{2}=0 \\
\text { or } \\
m=2, \quad a_{1}=0, \quad a_{2}=1, \quad b_{1}=b_{2}=\frac{1}{2}
\end{gathered}
$$

respectively, that is, the second numerical solution value is given by

$$
\begin{gathered}
u_{1}=e^{A t / 2} e^{B t} e^{A t / 2} u_{0} \\
\text { or } \\
u_{1}=e^{B t / 2} e^{A t} e^{B t / 2} u_{0}
\end{gathered}
$$

One of the most common and popularly used operator splitting method is the Strang splitting (or Strang-Marchuk operator splitting method). By the small adjustment in time step of length, it produced the strang splitting's algorithm. The idea is that instead of solving Equation (7) for a full time step length $\Delta t$, we solve the problem for a time step of length $\Delta t / 2$. We then solve the problem (8) for a full time step of length $\Delta t$, and finally (7) once more, again for a time interval of length $\Delta t / 2$. This gives the essential difference between Lie-Trotter and Strang spliting methods.

The Strang Splitting's algorithm is:

$$
\begin{gathered}
\frac{\partial u^{*}(t)}{\partial t}=A u(t), \text { with } t \in\left[t^{n}, t^{n+1 / 2}\right], u\left(t^{n}\right)=u_{s p}^{n} \\
\frac{\partial v(t)}{\partial t}=B v(t), \text { with } t \in\left[t^{n}, t^{n+1}\right], v\left(t^{n}\right)=u\left(t^{n+1 / 2}\right) \\
\frac{\partial w(t)}{\partial t}=A w(t), \text { with } t \in\left[t^{n+1 / 2}, t^{n+1}\right] \\
w\left(t^{n+1 / 2}\right)=v\left(t^{n+1}\right)
\end{gathered}
$$

where $t^{n+1 / 2}=t^{n}+0.5 \Delta t$, and the approximated split solution at the point $t=t^{n+1}$ is defined as $u_{s p}^{n+1}=w\left(t^{n+1}\right)$.

\section{Consistency of the Operator Split- ting Method}

Consider the linear ODE :

$$
\frac{d u}{d t}=A u
$$

Let $A=B+C$, then the splitting method for solving (14) is defined by solving first

$$
\frac{d u}{d t}=B u
$$

followed by solving

$$
\frac{d u}{d t}=C u
$$

For one step size $\Delta t$, we write the solution of (14) as $\Phi_{\Delta t}(u)=e^{A \Delta t}$ and the solution of (15)-(16) as $\Psi_{\Delta t}(u)=$ $e^{C \Delta t} e^{B \Delta t}$

The local error is:

$$
l e(u ; \Delta t)=\Phi_{\Delta t}(u)-\Psi_{\Delta t}(u)=e^{A \Delta t}-e^{C \Delta t} e^{B \Delta t}
$$

To estimate the local error, we use the exponential series, we have

$$
\begin{gathered}
l e(u ; \Delta t)=\left(1+A \Delta t+\frac{1}{2} A^{2} \Delta t^{2}+\ldots\right) \\
-\left(1+C \Delta t+\frac{1}{2} C^{2} \Delta t^{2}+\ldots\right)\left(1+B \Delta t+\frac{1}{2} B^{2} \Delta t^{2}+\ldots\right) \\
=\left(1+A \Delta t+\frac{1}{2} A^{2} \Delta t^{2}\right)-(1+\Delta t(C+B) \\
\left.+\Delta t^{2}\left(C B+\frac{1}{2} C^{2}+\frac{1}{2} B^{2}\right)+O\left(\Delta t^{3}\right)\right)
\end{gathered}
$$

Replacing $A$ by $B+C$ and noting that $A^{2}=B^{2}+B C+$ $C B+C^{2}$, (18) becomes

$$
\Delta t^{2}\left(\frac{1}{2}(C+B)^{2}-\left(C B+\frac{1}{2} C^{2}+\frac{1}{2} B^{2}\right)\right)+O\left(\Delta t^{3}\right)
$$




$$
\begin{aligned}
& =\Delta t^{2}\left(\frac{1}{2} C^{2}+\frac{1}{2} C B+\frac{1}{2} B C+\frac{1}{2} B^{2}-C B-\frac{1}{2} C^{2}-\frac{1}{2} B^{2}\right) \\
& +O\left(\Delta t^{3}\right) \\
& =\frac{1}{2} \Delta t^{2}(B C-C B)+O\left(\Delta t^{3}\right) \\
& =\frac{1}{2} \Delta t^{2}[B, C]+O\left(\Delta t^{3}\right)
\end{aligned}
$$

If the matrix $[B, C]=B C-C B=$ zero then the matrices $B$ and $C$ are commutative [8]. In such a case, it can be shown that not only the 2 nd order term in the local error vanishes, but furthermore,

$$
e^{B \Delta t} e^{C \Delta t}=e^{A \Delta t}
$$

\section{Stability of exponential splitting method}

Stability of exponential Lie and exponential Strang splitting methods have won a lot of attention in the recent years $[9,10,11]$. In this section, we look at the linear systems of ODEs and in particular at influence of perturbations at such systems.For doing this, we follow the work in [12]. Consider the initial value problem,

$$
\frac{d U}{d t}=A U(t), t \in[0, T], U(0)=U_{0}
$$

a given matrix $A \in \mathbb{R}^{m \times m}$. The solution of Equation (19)

$$
U(t)=e^{t A} U_{0}
$$

Consider also a perturbed problem,

$$
\frac{d \hat{U}}{d t}=A \hat{U}(t)+\delta(t), t \in[0, T], \hat{U}(0)=\hat{U}_{0}
$$

Then, for $\varepsilon(t)=\hat{U}(t)-U(t)$ we find by the variation of constant formula that,

$$
\varepsilon(t)=e^{t A} \varepsilon(0)+\int_{0}^{t} e^{(t-s) A} \delta(s) d s,
$$

which leads to the norm estimate

$$
\|\varepsilon(t)\| \leq\left\|e^{t A}\right\|\|\varepsilon(0)\|+\int_{0}^{t}\left\|e^{(t-s) A}\right\| d s \max _{0 \leq s \leq t}\|\delta(s)\|
$$

Consequently, if we have the following stability inequality

$$
\left\|e^{t A}\right\| \leq K e^{t w} \text { for all } t \geq 0
$$

with constants $K$ and $w \in \mathbb{R}$, then we obtain

$$
\|\varepsilon(t)\| \leq K e^{t w}\|\varepsilon(0)\|+\frac{K}{t}\left(e^{t w}-1\right) \max _{0 \leq s \leq t}\|\delta(s)\|
$$

with convention $\left(e^{t w}-1\right) / w=t$ in case $w=0$. This inequality shows that the overall error $\|\varepsilon(t)\|$ can be bounded in terms of the initial error $\|\varepsilon(0)\|$ and perturbations $\|\delta(s)\|$ , $0 \leq s \leq t$. The term stability will be used to indicate that small perturbations result in a small overall effect [13]. We now take a closer look at bounds for $\left\|e^{t A}\right\|$. Suppose that $A$ is diagonalizable, $A=P \Lambda P^{-1}$, where $\Lambda=\operatorname{diag}\left(\lambda_{k}\right)$ and that the vector norm is absolute. Then, $e^{t A}=P e^{t \Lambda} P^{-1}$, and

$$
\left\|e^{t A}\right\| \leq\|P\|\left\|e^{t \Lambda}\right\|\left\|P^{-1}\right\|=\operatorname{cond}(P) \max _{1 \leq k \leq m}\left|e^{t \lambda_{k}}\right|
$$

Consequently, if we know that $\operatorname{cond}(P)=\|P\|\left\|P^{-1}\right\| \leq K$ and $R e \lambda_{k} \leq w$, then

$$
w=\max _{1 \leq k \leq m}\left|e^{t \lambda_{k}}\right|
$$

In particular, if $\mathrm{A}$ is normal matrix, then the matrix of the eigenvectors $P$ is unitary. Since $e^{t A}=P e^{t \Lambda} P^{-1}$ the matrix $e^{t A}$ is also normal. Thus with the normal matrices $A$ we have

$$
\left\|e^{t A}\right\|_{2}=\max _{1 \leq k \leq m}\left|e^{t \lambda_{k}}\right|
$$

Assume that matrix $A$ in Equation (19) has two term splitting as follows:

$$
A=A_{1}+A_{2}
$$

Then, the solution of (19) is given by

$$
U\left(t_{n+1}\right)=e^{t A} U\left(t_{n}\right),
$$

where we partition the space $[0, T]$ by equally spaced points $t_{0}, \ldots, t_{N}$, where $N$ is a positive integer and $\Delta t=T / N$ and $t_{n+1}=t_{n}+\Delta t$, for $n=0, \ldots, N-1$. If we wish to use only $A_{1}$ and $A_{2}$ separately, instead of the full matrix $A$, then Equation (6) can be approximated by

$$
U\left(t_{n+1}\right)=e^{\Delta t A_{2}} e^{\Delta t A_{1}} U\left(t_{n}\right)
$$

and Equation (10) can be approximated by:

$$
U\left(t_{n+1}\right)=e^{A_{2} \Delta t / 2} e^{A_{1} \Delta t} e^{A_{2} \Delta t / 2} U\left(t_{n}\right)
$$

with $U_{n}$ approximating $U\left(t_{n}\right)$. With regard to stability, if we have $\left\|e^{t A_{k}}\right\| \leq 1, k=1,2$, then it follows trivially that $\left\|U_{n+1}\right\| \leq\left\|U_{n}\right\|$ for the splitting (4). General stability results under the weaker assumption that $\left\|e^{t A_{k}}\right\| \leq K$ for $0 \leq$ $t \leq T$ with a constant $K>1$ seem unknown. However, in practice the splitting appears to be stable provided that substeps themselves are stable. In general, if the matrix $A$ is not normal, an estimate of $\operatorname{cond}(U)$ in some suitable norm maybe difficult to obtain. For this reason, we will look for more general concept to obtain bounds for $\left\|e^{t A}\right\|$. A useful concept for stability results with non-normal matrices is the logarithmic norm of a matrix $A$ in $\mathbb{R}^{m \times m}$, defined as

$$
\mu(A)=\lim _{\Delta t \rightarrow 0} \frac{\|I+\Delta t A\|}{\Delta t}
$$

In terms of logarithmic matrix norms, $\left\|e^{t A_{k}}\right\| \leq 1$ means that $\mu\left(A_{k}\right) \leq 0$ This implies $\mu\left(A_{1}+A_{2}\right) \leq 0$ and therefore $\left\|e^{t\left(A_{1}+A_{2}\right)}\right\| \leq 1$, so the system will be stable. 
Table 1. Numerical errors of the Lie-Trotter splitting and Strang splitting with initial data $T=\pi$ and $N=2^{14}$.

\begin{tabular}{|c|c|c|c|c|}
\hline$N$ & Trotter-error & Convergence Rate & Strang-error & Convergence Rate \\
\hline 32 & $2.8481 \mathrm{e}-002$ & 0.9774 & $1.4293 \mathrm{e}-003$ & 2.0145 \\
64 & $1.4374 \mathrm{e}-002$ & 0.9865 & $3.5676 \mathrm{e}-004$ & 2.0023 \\
128 & $7.2220 \mathrm{e}-003$ & 0.9930 & $8.9099 \mathrm{e}-005$ & 2.0015 \\
256 & $3.6199 \mathrm{e}-003$ & 0.9964 & $2.2266 \mathrm{e}-005$ & 2.0006 \\
512 & $1.8122 \mathrm{e}-003$ & 0.9982 & $5.5654 \mathrm{e}-006$ & 2.0003 \\
1024 & $9.0669 \mathrm{e}-004$ & 0.9991 & $1.3912 \mathrm{e}-006$ & 2.0001 \\
2048 & $4.5349 \mathrm{e}-004$ & 0.9995 & $3.4780 \mathrm{e}-007$ & 2.0001 \\
4096 & $2.2678 \mathrm{e}-004$ & 0.9998 & $8.6947 \mathrm{e}-008$ & 2.0000 \\
8192 & $1.1340 \mathrm{e}-004$ & 0.9999 & $2.1737 \mathrm{e}-008$ & 2.0000 \\
16384 & $5.6702 \mathrm{e}-005$ & 0.9999 & $5.4338 \mathrm{e}-009$ & 2.0001 \\
\hline
\end{tabular}

\section{Results}

We apply second-order splitting (Strang splitting) with Lie-Trotter Splitting to the Matrix problem and compare the solutions. we consider the system of ordinary differential equations:

$$
\frac{d U}{d t}+A(U)=0, \quad U(0)=U_{0}
$$

We split A into two sub-matrices $A_{1}$ and $A_{2}$ and give an approximate solution based on the strang splitting:

$$
U(n \Delta t) \approx\left[e^{\left(\frac{-\Delta t}{2} A_{2}\right)} \circ e^{\left(-\Delta t A_{1}\right)} \circ e^{\left(\frac{-\Delta t}{2} A_{2}\right)}\right]^{n} U_{0}
$$

We consider the matrix A to be:

$\mathrm{A}=\left[\begin{array}{llll}1 & 2 ; & -2 & 2\end{array}\right]$

Show the matrix :

$\mathrm{A}=$

$\begin{array}{rr}1 & 2 \\ -2 & 2\end{array}$

with initial value:

$\mathrm{UO}=[1 ; 1]$

Split A into $A_{1}$ and $A_{2}$ Make a splitting so that the eigenvalues of the two new matrices are real. We choose

$\mathrm{A} 1=\left[\begin{array}{llll}0.5 & 0 ;-2 & 1\end{array}\right]$
$\mathrm{A} 2=\mathrm{A}-\mathrm{A} 1$

Then, matrices $A_{1}$ and $A_{2}$ become:

$$
\begin{aligned}
& \mathrm{A} 1= \\
& 0.5000 \quad 0 \\
& -2.0000 \quad 1.0000 \\
& \mathrm{~A} 2= \\
& 0.5000 \quad 2.0000 \\
& 0 \quad 1.0000
\end{aligned}
$$

Results are given in Figure 1. Next step we examine exactitude of the Strang splitting and Lie-Trotter splitting we reckon the approximate solution at time $T=\pi$ using $N=2^{14}$ splitting steps, where we partition the space $[0, \pi]$ by equally spaced points $t_{1}, \ldots, t_{N}$, and $\Delta t=\pi / N$ and $t_{n+1}=t_{n}+\Delta t$, for $n=0, \ldots, N-1$. and notify the comparison between Lie-Trotter splitting error and Strang splitting error against $N$ Figure 2.

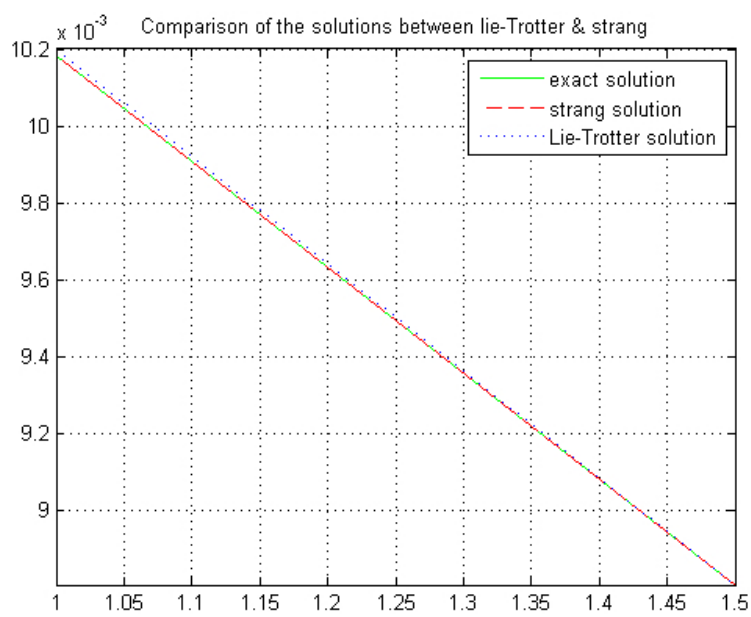

Figure 1. Comparison between Numerical solution of exponential LieTrotter splitting and exponential strang splitting for $\Delta t=0.0126$

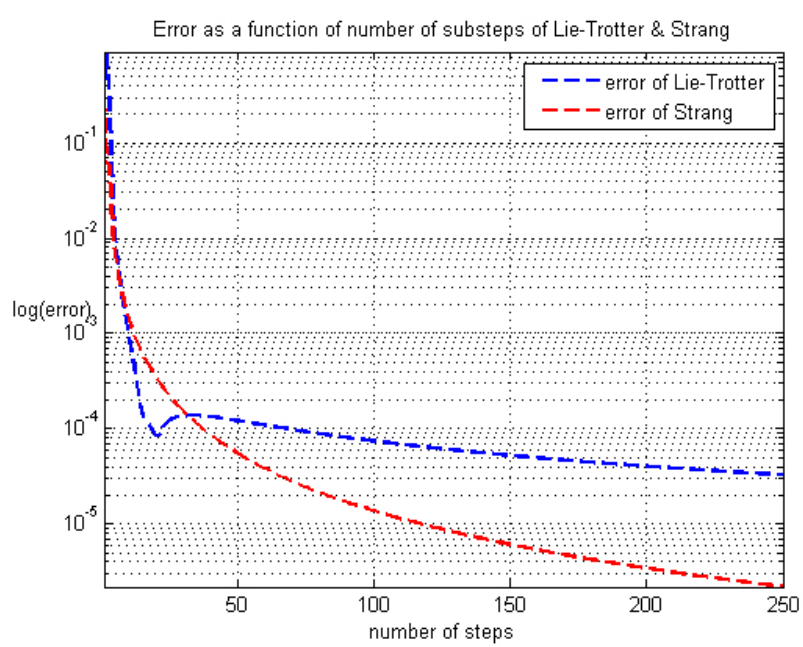

Figure 2. Comparison between Lie-Trotter errors splitting and starng splitting errors for $N=250$ 


\section{Conclusions}

This Paper discussed the Lie-Trotter and Strang splitting method for ODE system. The Strang method proved that the convergence happened with small data. The numerical solution is calculated up to $T=\pi$. We notice that for a small number of mesh points, the Strang splitting method has less errors than the Lie-Trotter splitting method. For large number of mesh points we notice from Figure 2 that the Strang splitting is much better than the Lie-Trotter splitting. Moreover Table 1 shows that the Lie-Trotter splitting method is of first order and Strang splitting method is of second order.

\section{REFERENCES}

[1] A. Bátkai, and P. Csomós, and G. Nickel. Operator splittings and spatial approximations for evolution equations. Journal of Evolution Equations, 9(3):613-636, 2009.

[2] F. Castella, P. Chartier, S. Descombes, and G. Vilmart. Splitting methods with complex times for parabolic equations. BIT Numerical Mathematics, 49(3):487-508, 2009.

[3] A. Chertock, Charles R Doering, E. Kashdan, and A. Kurganov. A fast explicit operator splitting method for passive scalar advection. Journal of Scientific Computing, 45(1-3):200-214, 2010.

[4] S. Descombes and M. Thalhammer. An exact local error representation of exponential operator splitting methods for evolutionary problems and applications to linear schrödinger equations in the semiclassical regime. BIT Numerical Mathematics, 50(4):729-749, 2010.

[5] I. Faragó and A. Havasi. Consistency analysis of operator splitting methods for $\mathrm{C} 0$-semigroups expression. In Semigroup Forum, pages 125-139. Springer, 2007.

[6] E. Hansenn and A. Ostermann. High order splitting methods for analytic semigroups exist. BIT Numerical Mathematics, 49(3):527-542, 2009.

[7] S. Blanes, F. Casas, and A. Murua. On the linear stability of splitting methods. Foundations of Computational Mathematics, 8(3):357-393, 2008.

[8] P. Csomós, I. Faragó, and Á. Havasi. Weighted sequential splittings and their analysis. Computers \& Mathematics with Applications, 50(7):1017-1031, 2005.
[9] E. Faou, A. Ostermann, and K. Schratz. Analysis of exponential splitting methods for inhomogeneous parabolic equations. IMA Journal of Numerical Analysis, 35(1):161-178, 2015.

[10] E. Hansen and A. Ostermann. Exponential splitting for unbounded operators. Mathematics of computation, 78(267):1485-1496, 2009.

[11] A. Ostermann and K. Schratz. Stability of exponential operator splitting methods for noncontractive semigroups. SIAM Journal on Numerical Analysis, 51(1):191-203, 2013.

[12] Y. Yazici. Operator splitting methods for differential equations. PhD thesis, Izmir Institute of Technology, 2010.

[13] Andrew Y. Ng, Michael I. Jordan, and Yair Weiss. On spectral clustering: Analysis and an algorithm. Advances in neural information processing systems, 2:849-856, 2002.

[14] P. Das and V. Mehrmann, Numerical solution of singularly perturbed convection-diffusion-reaction problems with two small parameters, BIT Numer. Math., 56, 51-76, 2016.

[15] P. Das, Comparison of a Priori and a Posteriori Meshes for Singularly Perturbed Nonlinear Parameterized Problems, J. Comput. Appl. Math., 290, 16-25,2015.

[16] P. Das and V. Mehrmann, Upwind based parameter uniform convergence analysis for two parametric parabolic convection diffusion problems by moving mesh methods, Proc. Appl. Math. Mech., 15, 591-592, 2015.

[17] P. Das and S. Natesan, Adaptive mesh generation for singularly perturbed fourth order ordinary differential equations, Int. J. Comput. Math., 92(3), 562-578, 2015.

[18] P. Das and S. Natesan, Richardson extrapolation method for singularly perturbed convection-diffusion problems on adaptively generated mesh, CMES Comput. Model. Eng. Sci 90, 463-485, 2013.

[19] P. Das and S. Natesan, Numerical Solution of a System of Singularly Perturbed Convection-Diffusion Boundary-Value Problems using Mesh Equidistribution Technique, Aust. J. Math. Anal. Appl., 10 (1), 1-17, 2013. 